\title{
A Study on Dual-Scale Data Charts
}

\author{
Petra Isenberg, Anastasia Bezerianos, Pierre Dragicevic, and Jean-Daniel Fekete
}

\begin{abstract}
We present the results of a user study that compares different ways of representing Dual-Scale data charts. DualScale charts incorporate two different data resolutions into one chart in order to emphasize data in regions of interest or to enable the comparison of data from distant regions. While some design guidelines exist for these types of charts, there is currently little empirical evidence on which to base their design. We fill this gap by discussing the design space of Dual-Scale cartesian-coordinate charts and by experimentally comparing the performance of different chart types with respect to elementary graphical perception tasks such as comparing lengths and distances. Our study suggests that cut-out charts which include collocated full context and focus are the best alternative, and that superimposed charts in which focus and context overlap on top of each other should be avoided.
\end{abstract}

Index Terms-Focus+Context, Quantitative Experiment, Dual-Scale Charts.

\section{INTRODUCTION}

Data charts such as line charts, bar charts, and scatter plots are arguably among the most common data representations used today. They have found widespread adoption in a variety of disciplines and are also frequently used to communicate information to the public. One of the problems of data charts in practice is that they become difficult to read when the amount of data goes beyond the available display resolution.

When the density of data points and/or their degree of interest is not uniform-e. g., time series with dense event clusters and large empty spaces in-between-one way to overcome visual resolution limitations is to use more than one scale (number of data units per display unit) in the same chart. Visually, this leads to charts where scale changes between regions with relatively high magnification, as well as regions where contextual data is displayed in a more condensed fashion.

Many different ways exist to visually integrate these different scales. Popular approaches include cut-out charts (Fig. 1(a)) and superimposed charts (Fig. 1(b)). Another common approach consists of changing the resolution along a single axis, i. e., applying a non-occluding step function [5]. In this case, Cleveland argues for the use of clear visual breaks-i.e., a split of the data chart into separate coordinate systems (Fig. 1(c)) - in order to indicate changes in the number of units per display unit [7, Chapter 2].

Apart from Cleveland's recommendation, little work exists to guide chart and visualization designers in choosing among different options for designing charts with more than one scale. In particular, empirical work is missing which highlights the advantages and disadvantages of existing approaches for elementary graphical perception tasks. Yet, tasks such as comparing distances and slopes in the data space are likely to be difficult when different scales are employed in the same chart, so choosing the right design seems of particular importance.

In this paper, we fill this gap by first discussing existing approaches and placing them within a common design space. We chose to focus on a specific subset of data charts where data points are positioned according to a two-dimensional orthogonal coordinate system. Examples of such charts include line charts, bar charts, area charts, and scatter plots but not pie charts, radar charts, 3-D charts, or organizational charts. Furthermore, we focus on charts with two different scales (Dual-Scale charts) which we believe are the most widespread,

- Petra Isenberg is with INRIA, E-mail: petra.isenberg@inria.fr.

- Anastasia Bezerianos is with École Centrale Paris (SAP BusinessObjects Chair) and LRI (Univ. Paris-Sud \& CNRS) \& INRIA E-mail: anastasia.bezerianos@ecp.fr.

- Pierre Dragicevic is with INRIA, E-mail: dragice@lri.fr.

- Jean-Daniel Fekete is with INRIA, E-Mail: jean-daniel.fekete@inria.fr.

Manuscript received 31 March 2011; accepted 1 August 2011; posted online 23 October 2011; mailed on 14 October 2011.

For information on obtaining reprints of this article, please send emailto:tvcg@computer.org. but still briefly discuss how the different approaches can be generalized to more than two scales. Although the term dual-scale has been often used to specifically refer to superimposed charts (Fig. 1(b)), in this article we use it to refer to any chart showing two main scales. We further present the results from a controlled user experiment which suggest that cut-out charts are the best option for Dual-Scale charts and that superimposed charts should generally be avoided. We conclude by providing general recommendations for choosing between different alternatives for Dual-Scale data charts

\section{BACKGROUND}

Our work relates specifically to past empirical work on the design of data charts as well as the larger field of focus-and-context presentation techniques. Throughout this paper we use the term "chart" to describe a graphical representation of data in which visual entities represent data points according to transformation functions ("rulers" [7]) associated with axes on a plane. We use "chart" instead of "graph" in order to avoid confusion with the larger field of graph drawing.

\subsection{Chart Design}

Many guidelines and design considerations exist for displaying data in the form of charts, e.g., [3, 7, 19, 29, 31]. While many early guidelines were based on authors' intuition drawn from practice [12], graphical perception experiments later on began to analyze and extend these early guidelines. Cleveland and Kosslyn provide comprehensive summaries of their empirical work on graphical perception studies as guidelines for chart construction [7, 19]. Studying the perception of charts is inherently important due to their widespread use in a variety of disciplines and popular media. Recent studies have investigated the effect of chart embellishments [2], elementary graphical perception tasks confirming and extending previous work [16], the use of charts and their annotations in Internet-sized collaborative work [32], or have investigated general graph reading and comprehension for education purposes $[12,26]$. Our work is most closely related to Heer and Bostock's recent study [16] as we also base our work on Cleveland's elementary graphical perception tasks. Yet, we consider a novel chart context which has not previously been a factor in perceptual studies of data charts. Our Dual-Scale chart context is closely related to focus-and-context techniques and studies thereof as outlined next.

\subsection{Focus-and-Context Techniques}

Focus-and-context techniques are related to our work in that they are methods for integrating regions of different scale into a single data representation. Several overview articles discuss varying taxonomies and software frameworks for focus-and-context displays $[6,9,18,20,23,24]$. Leung and Apperley [20] distinguish between distortion-oriented and non-distortion-oriented techniques. Distortionoriented techniques are often described as "lenses," "focus-in-context," 


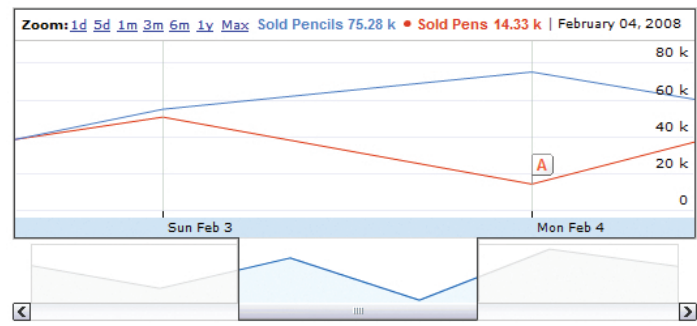

(a) A cutout chart as available through the Google Charts API [14]. The top part shows a subset of the data at a larger scale.

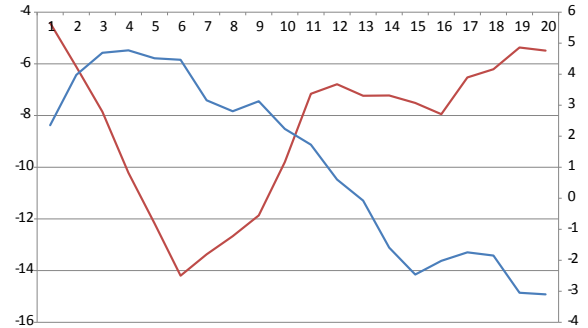

(b) A superimposed Microsoft Excel chart. The red line is plotted according to the left and the blue according to the right $y$-axis.
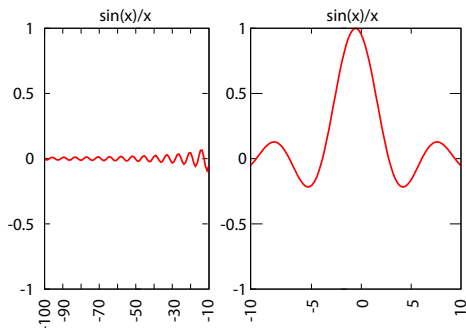

(c) A broken chart made with Gnuplot [35]. Left and right panels show the same function for different $x$-ranges.

Fig. 1. Three examples of Dual-Scale data charts made with common charting software.

or "multi-scale" and include such classic techniques as the bifocal display [30], the perspective wall [21], or the rubber sheet [28]. Nondistortion-oriented techniques include zooming and windowing techniques such as radar views. In this paper, we discuss both distortionoriented as well as non-distortion-oriented techniques with a focus on Dual-Scale changes along one chart axis. We discuss the most related previous focus-and-context techniques to our charts in Section 3.

Our work also relates to previous studies on the comprehension of focus-and-context views. Generally focus-in-context methods are believed to reduce cognitive effort needed to integrate contextual information compared to windowing or zooming techniques $[6,13,36]$. Cockburn et al. [9] summarize empirical studies which targeted comprehension issues with focus-and-context techniques in information spaces. Most of the past studies involved comprehension in the context of manipulation and interaction of a distorted and non-distorted information space. Relatively few studies have targeted visual perception independent of interaction. In this context, the most related work to ours is a study by Zanella et al. [36] on visual cues to aid comprehension of a visual distortion in an information space. A grid was found to be the most useful and preferred technique and we, thus, included grids as visual indicators of scale changes in all our charts in the study. In this paper, we contribute specifically to the literature on perception studies for focus-and-context displays, irrespective of the presence of interaction techniques. We provide an understanding on how well participants are able to relate information in focus-and-context regions in data charts depending on how they are visually integrated.

\subsection{Dual-Scale Techniques for Data Charts}

The specific context of our study is Dual-Scale data charts. These types of charts are available from commercial or free tools such as the Google Charts API, Microsoft Excel, or Gnuplot [35] as shown by the three examples in Fig. 1. We know of no empirical work which compares different Dual-Scale chart techniques, yet some recommendations on their use have been established by researchers and practitioners: In his "principles for graph design" [7], Cleveland contributes several recommendations relating to multi-scale charts. When comparing different charts he suggests to use the same data mappings if possible, common units per display unit when possible, and to make changes in data scale visually clear if they cannot be avoided. When a break in data scale is necessary, Cleveland suggests not to connect data values across the break. Similar recommendations were also made by Cox [10] in an earlier article. Several experts [11, 15, 29] discuss problems with the Dual-Scale chart in Fig. 1(b) and recommend careful design or to avoid using it altogether. Yet, empirical evidence on the effectiveness of the Dual-Scale compared to other charts is missing. In general, there is little empirical work on focus-and-context techniques specifically dedicated to data charts. Our work focuses on elementary graphical perception tasks for quantitative data as suggested by Cleveland $[7,8]$. Cleveland introduces ten tasks: among them length, position along one common scale, and slope all of which we also tested in our study. Extending this work, we also tested length, position, and slope for axes with two different data resolutions and along both in- terrupted and non-interrupted axes. Perhaps the most closely related study to our work in this space was conducted by Wigdor et al. [34] for the perception of graphical elements on a display set at varying viewing angles-from tabletop to upright. Their study found that error increased when graphical variables were compared with increasing distortion. Yet, in contrast to our work, they tested distortion as a factor of viewing angle, while we are interested in distortion as a factor of the graphical representation.

\section{Dual-Scale Data Charts}

In this section we first introduce our terminology for characterizing Dual-Scale charts. Then, we provide main examples in this design space and outline their properties, visual variants, and generalizations. This formal characterization is meant to provide a common framework for designing, discussing, and evaluating these types of charts.

\subsection{Terminology}

Before discussing the differences between Dual-Scale chart techniques, it is helpful to establish a set of common terms to describe them.

\subsubsection{Dual-Scale Transformation Functions}

A Dual-Scale chart technique can be characterized with the help of transformation functions as used by Leung and Apperley [20] in their taxonomy of distortion-oriented presentation techniques.

A transformation function $T$ maps a point $u \in D$ in data space to a point $u^{\prime} \in D^{\prime}$ in display space (e. g., in the drawing region of a chart):

$$
T: \quad D \rightarrow D^{\prime} ; \quad u \mapsto u^{\prime}
$$

Since we focus on charts where data points are positioned according to a $2 \mathrm{D}$ rectangular coordinates system, we consider the transformation function to be chosen per axis and assume that $T$ takes a single numerical parameter and returns a single numerical value.

The magnification function $M$ of the transformation function $T$ is its first derivative:

$$
M(u)=\frac{d u^{\prime}}{d u}
$$

This means that $M(u)$ represents the scale (number of data units per display unit) at the location $u^{\prime}=T(u)$ in display space.

Transformation functions and magnification functions can have various shapes. Among these, linear transformation functions-or constant magnification functions-are the most common. More complex transformation functions have been used such as logarithmic scales or fisheye distortions [6]. In this article, we are interested in DualScale transformation functions, which we define as follows: $T$ is a Dual-Scale transformation function if:

$$
\begin{array}{lll}
T(u)=T_{1}(u) & \text { if } & u \in D_{1} \\
T(u)=T_{2}(u) & \text { if } & u \in D_{2}
\end{array}
$$

and: 


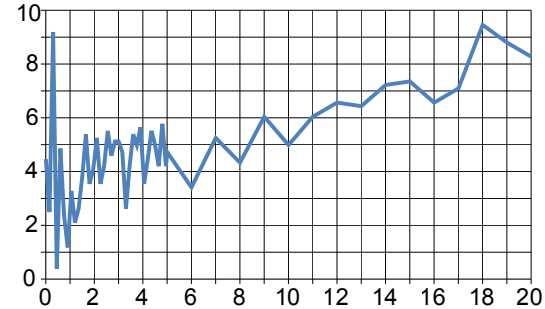

(a) A regular chart.

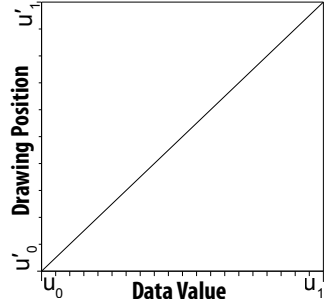

(b) $T$ for the $x$-axis.

Fig. 2. A regular, single-scale bivariate line chart (left) and its $x$-axis transformation function $T_{\text {single }}$ (right).

- $T_{1}$ and $T_{2}$ are monotonic linear functions with different slopes,

- $D_{1}$ and $D_{2}$ are intervals of $D$ verifying $D_{1} \cup D_{2}=D$ and $D_{1} \cap D_{2}=\emptyset$.

In other terms, a Dual-Scale transformation function partitions the dataset into two regions and applies a linear transformation to each of them. This yields a piecewise constant magnification function $M$ with two parts. In accordance with the terminology of focus-andcontext techniques, we call the part of highest magnification the focus region, and the part with the lowest magnification the context region. In addition, we call the transition point between these two regions the breakpoint $u_{b}$. We will occasionally use the notations $D=\left[u_{0}, u_{1}\right]$, $D_{1}=\left[u_{0}, u_{b}\right]$ and $D_{2}=\left[u_{b}, u_{1}\right]$, as well as $M_{1}$ (resp. $M_{2}$ ) for the magnification factor within $D_{1}$ (resp. $\left.D_{2}\right)$. Note that given this definition, a chart with one single logarithmic scale is not considered a Dual-Scale chart since it does not consist of two linear segments.

\subsubsection{Properties of Dual-Scale Transformation Functions}

We introduce simple mathematical properties that are useful for characterizing Dual-Scale transformation functions.

Most charts are based on transformation functions that are monotonic and minimally $C^{1}$ continuous (i. e., $T$ is differentiable and $M$ is continuous), and thus also bijective. This is the case for linear transformation functions, log scales, and most fish-eye transformations. As for Dual-Scale transformation functions, both $T_{1}$ and $T_{2}$ functions are minimally $C^{1}$ continuous (or simply $C^{1}$ ) but $T$ does not need to be so. In fact, there can be a corner or a discontinuity at the breakpoint, making the transformation function either $C^{0}$ ( $T$ is continuous but $M$ is discontinuous) or $C^{-1}$ ( $T$ is discontinuous).

We can, therefore, characterize three types of Dual-Scale transformation functions based on the order of continuity of $T$ and the resulting visual discontinuity that can be observed between $D_{1}^{\prime}$ and $D_{2}^{\prime}$. We name them following Carpendale's characterization [5] of visual connections between different regions in a presentation. They are:

visually disjoint if $T$ is $C^{-1}$, there is a visual gap between $D_{1}^{\prime}$ and $D_{2}^{\prime}$, visually continuous: if $T$ is minimally $C^{0}$, i. e., $D_{1}^{\prime}$ and $D_{2}^{\prime}$ are visually adjacent with possibly an abrupt change in scale, or visually integrated: if $T$ is minimally $C^{1}$, i.e., $D_{1}^{\prime}$ and $D_{2}^{\prime}$ are smoothly connected visually, without any abrupt change in scale.

Note that Carpendale also defines visually adjacent for regions which are side-by-side but whose content is possibly not aligned. One example for this case is the removal of parts of the data by choosing $D_{1}$ and $D_{2}$ so that they are not adjacent; a scale break approach as described by Cleveland [7]. We do not treat this case here and focus on the case $D_{1} \cup D_{2}=D$. The next section provides examples of DualScale transformation functions for common Dual-Scale techniques.

\subsection{Regular Charts}

A regular, single-scale data chart is illustrated in Fig. 2(a) as an example of reference. Here, the transformation function shown to the right of the figure is applied to the $x$-axis. The transformation function $T_{\text {single }}$ linearly maps the data domain $D=\left[u_{0}, u_{1}\right]$ (with $u_{0}=0$

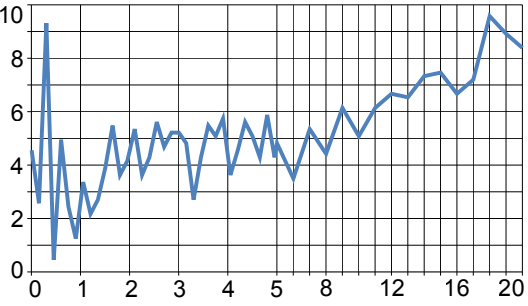

(a) A bifocal chart.

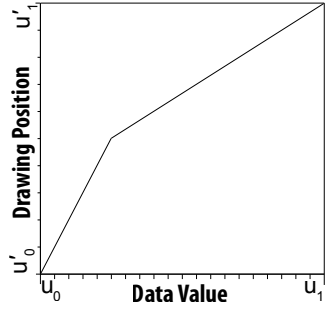

(b) $T$ for the $x$-axis.
Fig. 3. A bifocal line chart with a breakpoint at $u_{b}=5$ (left) and its $x$-axis transformation function $T_{\text {bifocal }}$ (right).

and $u_{1}=20$ in the figure) to the display domain $D^{\prime}=\left[u_{0}^{\prime}, u_{1}^{\prime}\right]$. Its corresponding magnification function is the constant $M_{\text {single }}=\left(u_{1}^{\prime}-\right.$ $\left.u_{0}^{\prime}\right) /\left(u_{1}-u_{0}\right) . T_{\text {single }}$ is a simple function with good properties (it is monotonic and $C^{\infty}$ ), but one problem is that function values for $u \in[0 ; 5]$ are dense and difficult to read.

In all the following examples, we assume that $D$ has already been partitioned into two regions $D_{1}$ and $D_{2}\left(u_{b}=5\right.$ on the figures), with $D_{1}$ being the focus and $D_{2}$ the context. This partitioning can either reflect the a priori importance of the data (possibly computed automatically from the data distribution) or a Degree of Interest (judged by a chart designer or controlled interactively by a computer user) [13].

\subsection{Bifocal Charts}

Fig. 3 shows a chart with two regions of different scales along the $x$ axis. Visually, this yields a variant of the Bifocal Display [30]. The focus region is enlarged to fill more drawing space and the remaining data is compressed into the remaining available space.

\section{PROPERTIES:}

Similar to the regular chart, the transformation function $T_{\text {bifocal }}$ (see Fig. 3(b)) is bijective and monotonic. However, it is $C^{0}$, which yields a visually continuous but visually non-integrated connection between the regions $D_{1}^{\prime}$ and $D_{2}^{\prime}$. This means that the chart is continuous but exhibits an abrupt change in scale, which can potentially make it difficult to cognitively relate information in both regions $[6,13,36]$.

\section{VARIANTS:}

The data breakpoint $u_{b}$ being already chosen and assuming that we choose the display domain $D^{\prime}$ to be the same as in the regular chart, the only remaining free parameter for this type of transformation function is the visual location of the breakpoint $u_{b}^{\prime}$ (in our example we chose $\left.u_{b}^{\prime}=\left(u_{0}^{\prime}+u_{1}^{\prime}\right) / 2\right)$. Once this location has been chosen, the transformation function is fully defined, and with it the magnification factors $M_{1}$ and $M_{2}$. In our example in Fig. 3, the relative magnification $M_{1} / M_{2}$ is 3. Alternatively, this relative magnification can be chosen as the free parameter, in which case it determines the value of $u_{b}^{\prime}$.

A visually integrated and a visually discontinuous version of the bifocal chart's transformation function are detailed in the next sections.

In terms of visual design, to make the scale transition visually more salient one can consider to add two vertical || or slanted / / lines on the axis representation to indicate a change in magnification. Cleveland calls this technique a partial scale break [7] but recommends to use a broken chart instead, as described in Section 3.5.

\section{Generalizations:}

Extending bifocal charts to support multiple scales is straightforward. By adding multiple "bends" in $T$, an arbitrary number of regions with different magnification factors can be created. The $1 \mathrm{D}$ distortion applied to bifocal charts can also be extended to 2D by applying it individually to each axis. This, however, may lead to ghost foci [5].

\subsection{Lens Charts}

Fig. 4 illustrates the effect of adding a drop-off function to the bifocal chart of Fig. 3, in a way similar to fish-eye lenses [27]. The scale is constant in the focus region, then starts to smoothly decrease in 


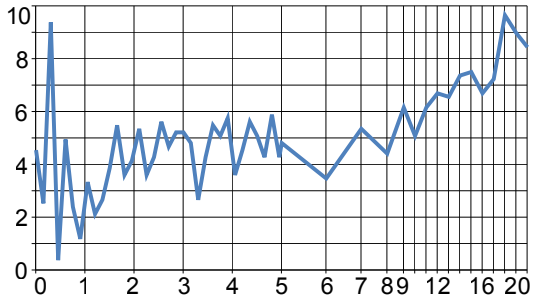

(a) A lens chart.

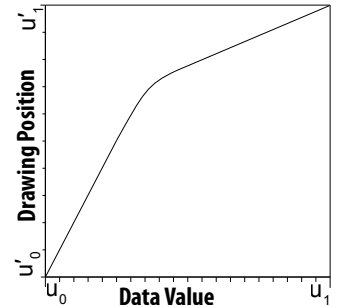

(b) $T$ for the $x$-axis.
Fig. 4. A lens chart with a drop-off region between $u_{b}=5$ and $u_{d}=9$ (left) and its $x$-axis transformation function $T_{\text {lens }}$.

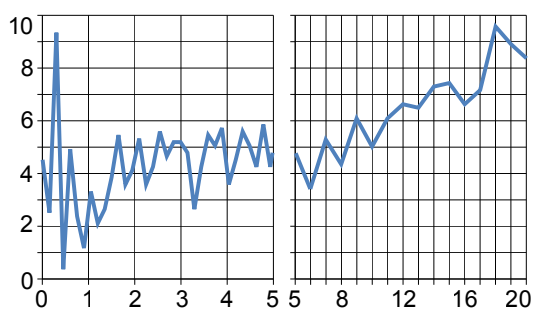

(a) A broken chart.

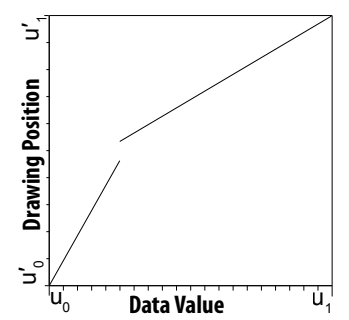

(b) $T$ for the $x$-axis.
Fig. 5. A broken chart showing a gap at $u=5$ (left) and its $x$-axis transformation function $T_{\text {broken }}$ (right).

the context region, then is constant again in the rest of the context region. Although this is technically not a Dual-Scale chart as previously defined in our terminology section, it does have two primary magnification factors and can therefore be classified into the family of Dual-Scale techniques provided the drop-off region is small enough.

\section{PROPERTIES:}

Like $T_{\text {bifocal }}$, the transformation function $T_{\text {lens }}$ (see Fig. 4(b)) is bijective and monotonic. However, it is $C^{1}$, which yields a visually integrated connection between regions $D_{1}^{\prime}$ and $D_{2}^{\prime}$, thanks to the drop-off function which bridges the gap between the two magnification factors.

\section{VARIANTS:}

The drop-off region can be chosen to overlap both the focus and the context regions, but we chose to fully preserve the focus region and have the drop-off encroach on $D_{2}$ instead. The data breakpoint $u_{b}$ being already chosen and assuming we choose the display domain $D^{\prime}$ and the breakpoint location $u_{b}^{\prime}$ to be the same as in the bifocal chart, the magnification factor $M_{2}$ necessarily has to be smaller (this is visible when comparing charts in Fig. 4 and Fig. 3).

There are an infinite number of possible drop-off functions and ways of parameterizing them. In the implementation we used in our user study, we chose to use as the unique parameter the visual location $u_{d}^{\prime} \in\left[u_{b}^{\prime}, u_{1}^{\prime}\right]$ of the transition point between the drop-off region and the constant-scale region $\left(u_{d}^{\prime}=9\right.$ in our example). The location $u_{d}$ of this transition point in the data space is chosen so that it verifies $\angle U_{0} U_{b} U_{d}=\angle U_{b} U_{d} U_{1}$, with $U=\left(u, u^{\prime}\right)$. We then interpolate between $U_{b}$ and $U_{d}$ using a quadratic Bézier curve. In our example of Fig. 4, this yields a relative magnification $M_{1} / M_{2}$ of 4.3 .

\section{GENERALIZATIONS:}

Lens charts can be extended to support multiple scales and 2D transformations the same way as bifocal charts.

\subsection{Broken Charts}

Fig. 5 shows an example of a broken chart. Such charts are promoted by Cleveland when several scales need to be shown [7] and are drawn with a visible gap between the regions with different scales and clear dividing lines on the circumference of the chart's data area.

\section{Properties:}

The transformation function $T_{\text {broken }}$ for this chart is monotonic but non-

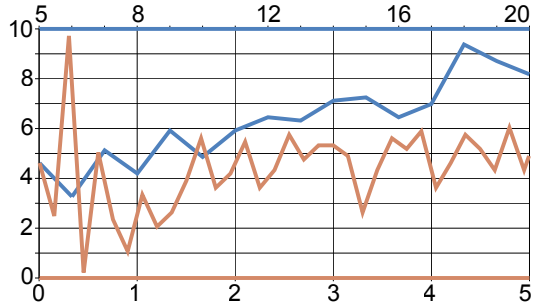

(a) A superimposed chart.

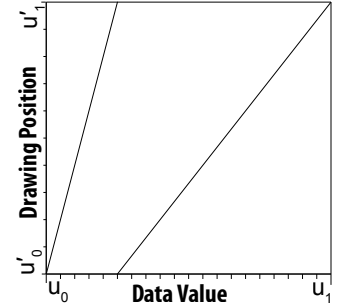

(b) $T$ for the $x$-axis
Fig. 6. A superimposed chart where focus and context are drawn on the same chart area (left), and its $x$-axis transformation function $T_{\text {sup }}$ (right).

bijective. It is injective but not surjective, which means that positions in the display domain encode data values unambiguously but not all these positions are effectively used. It is also discontinuous and has a gap whose size represents the amount of white space between the focus and context region. As a consequence, the focus and context regions are visually disjoint and their relationship has to be deduce from chart context such as labels [5].

\section{VARIANTS:}

We can choose to preserve either the focus region and have the gap encroach on the context, or to center the gap at the visual breakpoint. In our example we did the latter, which shrinks the context and focus regions slightly and yields slightly lower values for both $M_{1}$ and $M_{2}$. Once the gap placement strategy has been chosen, and choosing the same $u_{b}, D^{\prime}$ and $u_{b}^{\prime}$ as in the bifocal chart, the only remaining free parameter for $T_{b r o k e n}$ is the size of the gap $\Delta u_{g}^{\prime}$ in the display space.

In terms of visual design, a broken chart duplicates the orthogonal (y) axis after the gap where it can also hold tick marks and/or labels. As in Cleveland's original chart we only display tick marks (Fig. 5(a)).

\section{Generalizations:}

By introducing multiple breaks in the transformation function and varying the size of the gaps, more than one scale can be shown at a time. One issue is that gaps take screen real-estate so the more scales are shown, the less space remains for displaying the data. Broken charts also naturally extend to 2D. If one axis is partitioned into $m$ different scales and the other axis is partitioned into $n$ scales, the chart will show a total of $m \times n$ rectangular regions but, as for extensions to the bifocal chart, ghost foci may be created [5].

\subsection{Superimposed Charts}

Fig. 6 shows an example of a superimposed chart. Here both focus and context regions are drawn along the full width of the chart and share the same $y$-axis. Superimposed charts are commonly used when two data sets have only one axis in common.

\section{PROPERTIES:}

In contrast to previous chart types, the transformation function $T_{\text {sup }}$ for this chart is non-monotonic: data ordering is not preserved on the display space and simple ordinal comparisons might be more difficult. Also, the function is non-bijective but contrary to $T_{\text {broken }}$, it is surjective but not injective: it means that the whole display space is used but the encoding of data values is ambiguous. This might cause overlapping and legibility problems, although in some cases it could facilitate comparisons. In addition, $T_{\text {sup }}$ is $C^{-1}$ and thus the visual connection between focus and context is discontinuous. The size of this discontinuity $\left(u_{1}^{\prime}-u_{0}^{\prime}\right)$ is much larger than in the broken chart $\left(\Delta u_{g}^{\prime}\right)$.

This type of chart however has the advantage of having more resolution than the previous charts. When $u_{b}^{\prime}=\left(u_{0}^{\prime}+u_{1}^{\prime}\right) / 2$ as in our examples, the gain is twofold: for the same footprint $D^{\prime}$, the magnification factors $M_{1}$ and $M_{2}$ are doubled. Conversely, keeping the same magnification factors yields a chart twice as small.

VARIANTS:

In contrast with previous transformation functions, $T_{\text {sup }}$ does not take a parameter $u_{b}^{\prime}$ : the breakpoint is displayed both at $u_{1}^{\prime}$ and at $u_{0}^{\prime}$ because 


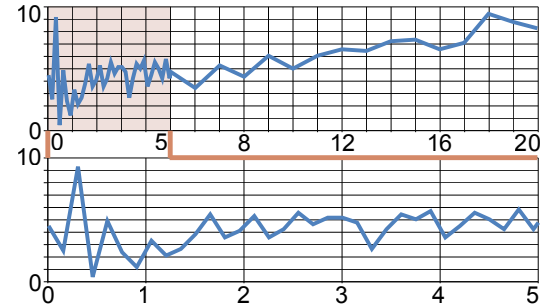

(a) A cut-out chart.

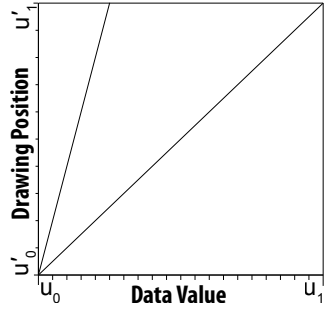

(b) $T$ for the $x$-axis.
Fig. 7. A cut-out chart where the focus between $u=0$ and $u=5$ is split off and shown separately (left) and the two transformation functions for its $x$-axis $T_{\text {cutout } 1}$ and $T_{\text {cutout } 2}$ (right).

this is what yields the best usage of the display space. Assuming we chose the same $u_{b}, D$ and $D^{\prime}$ as in the previous examples, $T_{\text {sup }}$ is fully determined and has no remaining free parameter.

Contrary to the bifocal approach, $T_{\text {sup }}$ cannot be reasonably turned into a smoother function. Although it is technically possible to insert a region around $u_{b}$ where the function smoothly decreases from $u_{1}^{\prime}$ to $u_{0}^{\prime}$, the visual result would probably make little sense.

The superimposed chart leaves the transformation function of the orthogonal axis ( $y$ in our examples) unchanged with respect to the regular chart. There are design alternatives where the data is drawn differently along this dimension. These include displaying focus and context "side-by-side" along the orthogonal dimension at the expense of footprint or resolution. In this case, the $2 \mathrm{D}$ transformation function becomes bijective and overlapping issues vanish, but ordering problems persist and the connection between regions remains discontinuous.

A superimposed chart typically displays axes from the the two datasets on both sides of the chart, and uses different colors or data point shapes for displaying the context and the focus data (see Fig. 6). Grid lines can also be displayed in two colors but for the sake of legibility, we chose to only display grid lines for the focus as the region of primary interest. Tickmarks were displayed for both regions.

\section{GenERALIZATIONS:}

Extending superimposed charts to more than two scales is straightforward as far as the transformation function is concerned. It involves partitioning $D$ into multiple regions and linearly mapping each of them to $D^{\prime}$. As in the Dual-Scale case, there is no remaining free parameter and the magnification factor of each region $\left[u_{n}, u_{n+1}\right]$ is fully determined and equal to $\left(u_{1}^{\prime}-u_{0}^{\prime}\right) /\left(u_{n+1}-u_{n}\right)$. This gives the superimposed chart approach less flexibility than the previous approaches as it is not possible to, e. g., shrink small regions. Drawing all sub-axes and showing how they map to the data points, however, is less straightforward. One approach could be to draw all axes side-by-side and use a color coding scheme but one would quickly run out of colors. An alternative would be to use a numbering scheme.

Extending superimposed charts to $2 \mathrm{D}$ is less trivial than with the previous techniques and applying transformation functions of the type $T_{\text {sub }}$ to both axes might yield visual representations that are difficult to read and interpret. This is an interesting topic for future work.

\subsection{Cut-Out Charts}

Fig. 7 shows an example of a cut-out chart. This type of chart is frequently used to display data along timelines [1]. The top part of the chart shows the whole dataset and highlights the focus while the bottom shows the focus extended to the width of the display area.

Since the focus region is shown twice, the transformation "function" $T_{\text {cutout }}$ for this chart is no longer a mathematical function. We thus either have to generalize the transformation function to a transformation mapping or allow several transformations functions to be applied in sequence. Fig. 7(b) shows these two functions $T_{\text {cutout } 1}$ and $T_{\text {cutout } 2}$ : $T_{\text {cutout } 1}$ linearly maps the focus region $D_{1}$ to $D^{\prime}$ (top line) and $T_{\text {cutout } 2}$ linearly maps $D$ to $D^{\prime}$ (bottom line). Cut-out charts can also affect the transformation function of the orthogonal axis by shrinking their display domain in order to show the two collocated plots.
PROPERTIES:

Both $T_{\text {cutout } 1}$ and $T_{\text {cutout } 2}$ are linear so, like in a regular chart, they are bijective, monotonic and $C^{\infty}$. In the top part of the chart the visual connection between the focus and context is visually integrated. So when these two parts are read like separate charts, they share all the properties of a regular chart.

When $T_{\text {cutout } 1}$ and $T_{\text {cutout } 2}$ are taken together (i. e., combined into a single mapping $T_{\text {cutout }}$ ) they are not injective anymore, but when considering the 2D transformation that combines the two axes, the mapping is bijective thanks to the side-by-side layout. This means that a $2 \mathrm{D}$ position on the chart unambiguously reflects a $2 \mathrm{D}$ data value, but when the user's visual attention switches between the two parts of the chart (e.g., reading data points in sequence on the magnified view then proceeding to the top view), the two regions appear as visually discontinuous and the whole chart as non-monotonic. This can make it potentially difficult to switch focus between the two parts of the chart and search for the same data point.

This chart has a good visual resolution along the $x$-axis, with the focus part having the same magnification as the superimposed chart (and better than all other charts) and the context part having the same magnification as the regular chart (better than all other charts except the superimposed one). Along the $y$-axis, however, the chart has a smaller magnification factor for the same footprint.

\section{VARIANTS:}

Like in the superimposed chart, $T_{\text {cutout }}$, and with it the magnification factors, are fully determined when $u_{b}$ and $D^{\prime}$ have been chosen.

In terms of visual design, the $x$-axis is typically duplicated on the two parts of the chart and additional visual cues make the relationship between the two views of the focus region explicit. The design space for these visual cues is large. Typical examples include displaying the focus and context regions on the top view with a different background color, and visually connecting the two views using lines or by enclosing them with a single shape (see Fig. 7 and Fig. 1).

\section{Generalizations:}

Cut-out charts can be extended to support multiple scales with some restrictions. For example, one could identify several focus regions in $D$ and display them side-by-side in the bottom part of the chart: instead of showing a single focus view, this region would then split its horizontal space among several focused views. This would, however, imply that the same scale is applied to all the other (non-focused) regions so that they can be shown in the top part. More flexible approaches can be thought of, such as using a hierarchical layout for focus regions as in [17]. Despite promising approaches, a method for automatically displaying cut-out charts with arbitrary partitionings of the data set and arbitrary scaling factors has yet to be found.

A straightforward extension of Dual-Scale cut-out charts to 2D consists in magnifying and cropping the $y$-axis in the focus view in addition to the $x$-axis, and displaying a "zooming rectangle" inside the context view. Furthermore, when the focus view is large enough (e. g., spans the whole screen), it is possible to place the context view as an inset into the focus rather than above it. This yields a 2D radar view, a technique that is often used in maps and graphic authoring tools to provide context and allow users to quickly pan a large document.

\subsection{Summary}

In this section we have provided representative examples of different types of Dual-Scale charts. These representative examples are distinguished by a number of properties we discussed throughout this section and summarize in Table 1.

The five first rows indicate the mathematical properties of the transformation function for the $x$-axis. The $x$ - and $y$-resolution rows indicate the amount of resolution available on these axes compared to the regular chart. scale DOF indicates the number of degrees of freedom available for choosing scales (i. e., $M_{1}$ and $M_{2}$ ) assuming the data breakpoint $u_{b}$ and the chart size $u_{1}^{\prime}-u_{0}^{\prime}$ are fixed. A value of 1 indicates that choosing a scale value determines the other (or similary, that only $M_{1} / M_{2}$ can be chosen). A value of 0 indicates that scales are imposed by the chart. 


\begin{tabular}{|c|c|c|c|c|c|c|c|}
\hline $\begin{array}{r}\text { CHART } \\
\text { /PROPERTY }\end{array}$ & (regular) & $\begin{array}{c}\text { bi- } \\
\text { focal }\end{array}$ & lens & broken & $\begin{array}{c}\text { super- } \\
\text { imposed }\end{array}$ & $\begin{array}{r}\text { cut- } \\
\text { per chart }\end{array}$ & $\begin{array}{l}\text { out } \\
\text { overall }\end{array}$ \\
\hline bijective $T$ & $\checkmark$ & $\checkmark$ & $\checkmark$ & - & - & $\checkmark$ & - \\
\hline injective $T$ & $\checkmark$ & $\checkmark$ & $\checkmark$ & $\checkmark$ & - & $\checkmark$ & - \\
\hline surjective $T$ & $\checkmark$ & $\checkmark$ & $\checkmark$ & - & $\checkmark$ & $\checkmark$ & $\checkmark$ \\
\hline monotonic $T$ & $\checkmark$ & $\checkmark$ & $\checkmark$ & $\checkmark$ & - & $\checkmark$ & - \\
\hline continuous $T$ & $C^{\infty}$ & $C^{0}$ & $C^{1}$ & - & - & $C^{\infty}$ & - \\
\hline$x$-resolution & $1 \times$ & $1 \times$ & $1 \times$ & $\approx 1 \times$ & $2 \times$ & $1-2 \times$ & $1-2 \times$ \\
\hline$y$-resolution & $1 \times$ & $1 \times$ & $1 \times$ & $1 \times$ & $1 \times$ & $0.5 \times$ & $0.5 \times$ \\
\hline scale DOF & & 1 & 1 & 1 & 0 & 0 & 0 \\
\hline
\end{tabular}

Table 1. Properties of representative examples of Dual-Scale charts.

After having characterized these representative examples of DualScale charts and their properties, the remaining essential question pertains to the effectiveness of each alternative Dual-Scale data encoding. We, therefore, designed an experiment to test chart performance for tasks in which data had to be compared across two scales.

\section{Experiment Design}

The purpose of our experiment was to determine how well different representations of Dual-Scale charts would perform for elementary graphical perception tasks. We were specifically interested in tasks which involve the comparison of data within the focus as well as across regions. We based this choice on the assumption that focus regions in charts are generally chosen to display data of importance but that this data often has to be put into context involving data outside the focus. The influence of chart representation and data scale for these tasks has not been previously addressed in the literature as outlined in Section 2.

\subsection{Experiment Factors}

Our main experimental factors were chart, task, location, and breakpoint. The details for each factor are outlined next.

\subsubsection{Charts}

We tested five charts for which we varied the scale along the $x$-axis. We describe them using the notations we introduced in Section 3. The horizontal display size $u_{1}^{\prime}-u_{0}^{\prime}=1200 p x$ was the same for all charts. We used $D=[0,20]$ for the $x$-axis and $D=[0,10]$ for the $y$-axis. We displayed a data grid on the $x$ - and $y$-axis showing one gridline for each integer data value in order to provide the same number of gridlines per representation. No labels were displayed for the charts. We chose the following charts to cover a range of Dual-Scale chart techniques:

Broken (B): A chart with a $T_{\text {broken }}$ transformation function (Fig. 5) whose visual breakpoint was set to $u_{b}^{\prime}=\left(u_{0}^{\prime}+u_{1}^{\prime}\right) / 2$ and whose gap size was set to $\Delta u_{g}^{\prime}=20 p x$ so that the focus and context regions covered just under $50 \%$ of the drawing space.

Cut-Out $(C)$ : A chart with a $T_{\text {cutout }}$ transformation function (Fig. 7). This chart was displayed with the same $y$-axis length for focus and context regions compared to the other four charts in order to avoid confounding effects due to $y$-axis distortion.

Lens $(L)$ : A chart with a $T_{\text {lens }}$ transformation function (Fig. 4). We set the visual breakpoint to $u_{b}^{\prime}=\left(u_{0}^{\prime}+u_{1}^{\prime}\right) / 2$ and set $u_{d}^{\prime}=\left(u_{b}^{\prime}+\right.$ $\left.u_{1}^{\prime}\right) / 2$ so that the drop-off region covered half of the context.

Regular $(R)$ : A regular chart with a $T_{\text {single }}$ transformation function (Fig. 2). This chart was chosen as a baseline comparison.

Superimposed $(S)$ : A chart with a $T_{\text {sup }}$ transformation function (Fig. 6). As shown in the figure, we used different data symbols or line colors for data in the focus and in the context.

\subsubsection{Tasks}

We used a subset of Cleveland's [7] elementary graphical perception tasks as we were interested in how information could be understood at a relatively quick glance across different data scales. We chose position, length, and slope tasks as the most highly ranked among Clevelands tasks [7] and also because we hypothesized them to be most impacted by changes in scale. Cleveland's past work [7] and later uses of his tasks (e.g., $[16,34])$ tested same-scale data along aligned or non-aligned axes. We extend this work by testing Dual-Scale data along aligned (lens) or non-aligned axes (broken, superimposed, cut$o u t)$. To do so, we asked participants to judge the position, length, and slope of the encoded information in data space rather than in drawing space. This meant that to answer questions correctly participants had to take gridline and tickmark spacing into account. For each task, we presented a modulus object on the chart and asked participants to judge the relative magnitude of a stimulus object as a percentage of the modulus. Similar to Wigdor et al.'s work [34] we were interested in the change in perception between different conditions and, thus, worked with the perceived magnitudes reported by the participants rather than modeling actual magnitudes as done by others [22]. For all tasks, the modulus value to judge was larger than the stimulus and, therefore, answers were always between 0 and $100 \%$. The modulus and stimulus were placed on different $y$-positions to make them distinguishable. For all conditions, the positions, lengths, and slopes were drawn to be fully visible in both the focus as well as the context as we were not interested in studying visual acuity [33]. Instead, we wanted to understand how-given visible data-participants were able to understand and relate the data presented to them at different scales.

\subsubsection{Location}

We tested three combinations of modulus and stimulus location:

Focus: Both stimulus and modulus are placed in the context region. This location was a control condition and was treated separately in the analysis of the data.

Focus +Context: The modulus is placed in the context while the stimulus is placed in the focus.

Across: The modulus and stimulus cross the scale break. This condition was only tested for length and slope tasks. For the position task only single data points were placed which have no extent and, thus, cannot cross $u_{b}$.

We used two slightly varied positions for the modulus in each location to avoid learning effects.

\subsubsection{Breakpoints}

We tested three different breakpoints which determined the overall scale in context and focus region as outlined in Section 3. The breakpoints were located at $u_{b}=2, u_{b}=5$, and $u_{b}=10 . u_{b}=10$ was a control condition, as there was no scale difference between focus and context except for the cut-out chart where the focus was scaled $2 \times$ compared to the context.

\subsection{Hypotheses}

Our hypotheses for this experiment were as follows:

$H_{1} \quad$ The chart performance is affected by factors location and breakpoint. Results are more prominent for higher scale differences. This hypothesis follows from previous work on perceptual distortion for elementary graphical perception tasks [34].

$\mathrm{H}_{2} \quad \mathrm{R}$ outperforms all charts for across and focus + context locations for all tasks. This is due to the uniform scale across all areas of this chart. We hypothesize a uniform scale to be significantly less cognitively demanding.

$\mathrm{H}_{3} \quad$ For location focus all techniques perform similarly for each task in terms of error, with possible small effects in time. Results follow those derived by Cleveland \& McGill [8]. This hypothesis is a direct follow-up from $\mathrm{H}_{2}$ as there exists a uniform scale within the focus region.

$H_{4} \quad L$ outperforms $B$ due to the smooth continuous visual integration of the context. This hypothesis results from focus-in-context techniques generally being believed to be less cognitively demanding $[6,13,36]$. 
We were less sure about the performance of $S$. On the one hand its $x$-resolution is high, on the other the chart is non-monotonic, its $T$ is $C^{-1}$, and focus and context are visually discontinuous. The benefits of the resolution may be outweighed by the added cognitive demand of having to select which axis to related visual entities to.

\subsection{Overall Experiment Design}

We used a repeated-measures design with the independent variables as outlined in Section 4.1. The dependent variables were magnitude of error and time. Each participant performed six trials per task $\times$ chart $\times$ location $\times$ breakpoint combination. The order of chart, task, location, and breakpoint was randomized using a Latin square. Each trial presented the data using a different percentage of change. The percentages were always multiples of 10 ranging from 10-60 for the position task and 20-70 for the length and slope tasks. The presentation order of the percentages was randomized for each set of six trials to minimize learning effects. Before each task, participants went through a set of training trials not used in the actual experiment, in which they saw each chart and location condition using one breakpoint. We did not provide participants with correct answers during the training to avoid learning correct answers. The experimental session lasted between 90 to 120 mins overall. In summary, the design included:

\begin{tabular}{rlr}
3 & tasks & $\times$ \\
5 & charts & $\times$ \\
$2 / 3 / 3$ & locations & $\times$ \\
3 & breakpoints & $\times$ \\
6 & percentages & $=$ \\
\hline 720 & trials per participant & $\times$ \\
15 & participants & $=$ \\
\hline $\mathbf{1 0 , 8 0 0}$ & trials in total &
\end{tabular}

\subsection{Participants and Procedure}

Fifteen participants (10 male, 5 female) were recruited from our research institute. Participants ranged from 24-39 years in age (median age 26) and all reported normal or corrected-to-normal vision. Eight participants were students and seven non-students with predominantly technical occupations. Nine participants reported at least weekly exposure to charts like the ones seen in the study; the remaining six participants reported monthly or less frequent exposure. Participants received instructions on how to read all charts and were not paid for their involvement in the study.

Participants sat in front of a 20.1 inch Dell LCD display at a distance of approximately $50 \mathrm{~cm}$. Participants answered all trials by entering the answer on the keyboard and then pressing the Enter key. Timing was started once a chart appeared on the screen and stopped when a participant hit the first number key to enter an answer. After a number key had been hit, the current chart was made invisible. This was done to ensure that we measured the time until an answer had been decided upon and not the typing time. After the study, participants filled out a questionnaire eliciting demographic information as well as subjective preferences for the different chart types tested.

\section{RESULTS}

Our dependent variables were time and error with error defined as previously suggested [8] as $\log _{2}\left(\mid j u d g e d\right.$ percent - true percent $\left.\mid+\frac{1}{8}\right)$.

This metric calculates the absolute difference between the user's judgement and the true magnitude difference. The $\log _{2}$ parameter was found appropriate for error judgments, while the $\frac{1}{8}$ parameter prevents a distortion of the results towards the lower end of the error scale, since the absolute error was sometimes close to 0 . Trials were marked as outliers when error was beyond two standard deviations from the mean for a given task, chart, location, breakpoint, and answer percent (4\% of all trials), and were removed from further analysis. The remaining trials were aggregated per subject for each combination of conditions, and followed the normal distribution. We also examined our participants' speed in performing the different tasks for charts, to look for potential time - error trade-offs. All analyses of the study data were performed using an ANOVA.

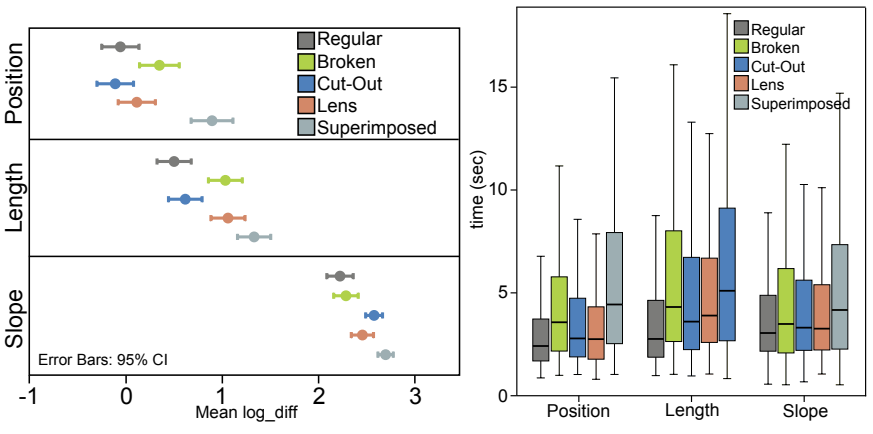

Fig. 8. Results for error (left) and time (right) per task for each chart.

We first compared performance across the three tasks (Fig. 8): We compared the common percents $(20,30,40,50,60)$ and common locations (focus, focus + context) across tasks. We found a significant effect of task on error $\left(F_{2,28}=166, p<.0001\right)$. Pair-wise means comparison (all $p<.05$, all adjustments Bonferroni) showed that users were significantly more accurate for the position task $(6.5 \%$ mean absolute distance error before transform), followed by length $(8.1 \%)$ and slope $(16 \%)$. There was no significant effect on time. Thus task accuracy in our experiment follows the order reported by Cleveland \& McGill [8] $\left(\mathrm{H}_{3}\right)$, not only for focus but also focus + context locations.

We found a significant effect of location $\left(F_{1,14}=70, p<.0001\right)$ on error, with tasks in focus being significantly more accurate $(p<.05)$, supporting $H 1$ that location affects accuracy. Post-hoc comparisons showed no significant difference for our control condition focus between charts in terms of error or time. The lack of significance and the very close means for all charts per task supports $H 3$. Thus, in the results reported below we focus on the remaining locations.

Finally, there was a significant effect of breakpoint $\left(F_{2,28}=29, p<\right.$ $.0001)$ on error. For all tasks, responses where significantly more accurate in the $u_{b}=10$ breakpoint, followed by $u_{b}=5$, and $u_{b}=$ 2 ; this supports $H 1$, that tasks are harder with an increase in scale difference. Next, we discuss our results independently for each task.

\subsection{Position}

The position task varied from the other two tasks in that we could not test the across location (Section 4). Results for time and error on the focus + context location are reported next. Fig. 9(a) gives a summary of the significant effects for this task and the charts tested.

Error: We found a significant effect of $\operatorname{chart}\left(F_{4.56}=25, p<.0001\right)$ on error. Pair-wise means comparison (all $p<.05$ ) showed that $R$ was significantly more accurate than all other charts except for $C$, and all charts were more accurate than $S$. Mean absolute error was -.2 for $R$ (close to 0 magnitude error), -.07 for $C$ (similarly), .26 for $L, .95$ for $B$, and 2.1 for $S$. There was also a significant effect of breakpoint $\left(F_{2,28}=\right.$ $5.8, p<.05)$, with participants being significantly less accurate in the breakpoint $u_{b}=2(p<.05)$

Time: We found a significant effect of chart on time $\left(F_{4,56}=19.8\right.$, $p<.0001$ ). Pair-wise means comparison (all $p<.05$ ) showed that $R$ was significantly faster than all other charts apart from $C, C$ was significantly faster than $B$ and $S$, and all charts were significantly faster than $S$. Mean times were faster for $R(3.2 \mathrm{~s})$, followed by $C(4 \mathrm{~s}), L$ (4.6 s), $B(6 \mathrm{~s})$, and $S(8.1 \mathrm{~s})$.

\subsection{Length}

For the length task we first analyzed overall effects and then looked at interactions for the two locations tested as well as the three breakpoints. Fig. 9(b) gives a summary of the overall significant effects for this task and the different charts tested.

Error: ANOVA showed a significant effect of chart on error $\left(F_{4,56}=52.3, p<.0001\right)$. Pair-wise means comparison (all $\left.p<.05\right)$ showed that $R$ and $C$ were significantly more accurate than all other 


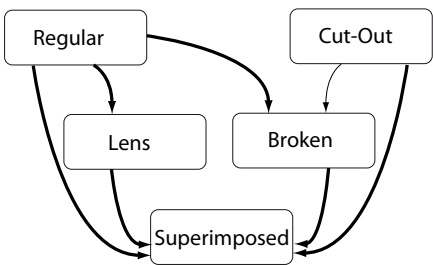

(a) Significant results between charts for the Position task.

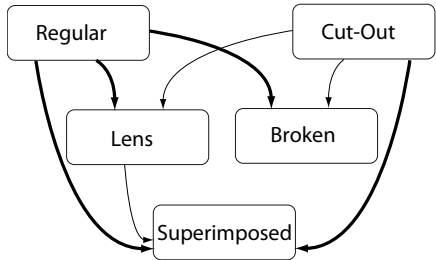

(b) Significant results between charts for the Length task.

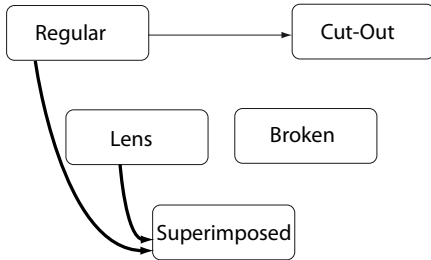

(c) Significant results between charts for the Slope task.

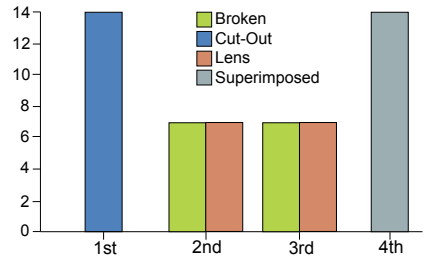

(d) Subjective ranking for the four Dual-Scale techniques.

Fig. 9. Significant interactions between chart for error and time for the three tasks in the study (Figures 9(a)-9(c)). Arrows indicate a significantly better than relationship. Thicker errors indicate that the effects were present for both error and time. Fig. 9(d) shows the overall main subjective ranking participants gave to each chart for each task. The rankings mirror the statistical results.

charts, and $S$ was less accurate than all charts apart from $B$. Mean absolute error was .3, .5, 1.09, 1.1,1.6 respectively for $R, C, B, L, S$.

There was also a significant effect of breakpoint $\left(F_{2.28}=9.6, p<\right.$ $.05)$, with participants being significantly more accurate for breakpoint $u_{b}=10(p<.05)$. A significant chart $\times$ breakpoint interaction was present $\left(F_{8,112}=7.1, p<.05\right)$. Post-hoc comparisons showed that for $u_{b}=2$ both $R$ and $C$ had significantly smaller error than all other charts. For $u_{b}=5$ we found that $S$ had the highest error among all charts, but $R$ and $C$ were no longer different from the remaining charts There were no differences between charts for $u_{b}=10$. There was also no effect of location on error.

Time: There was a significant effect of chart on time $\left(F_{4,56}=11\right.$, $p<.0001$ ). Pair-wise means comparison (all $p<.05$ ) showed that $R$ was significantly faster than all other charts apart from $C, C$ was faster than $S$. Mean times were fastest for $R(3.9 \mathrm{~s})$, followed by $C(5.2 \mathrm{~s}), L$ $(6 \mathrm{~s}), B(7.6 \mathrm{~s})$, and $S(8.4 \mathrm{~s})$.

\subsection{Slope}

The slope task was analyzed the same way as the length task. Fig. 9(c) gives a summary of the overall significant effects for this task between the different charts tested.

Error: The ANOVA showed a significant effect of chart on error $\left(F_{4,56}=4.7, p<.05\right)$. Pair-wise means comparison (all $p<.05$ ) showed that $R$ was significantly more accurate than $C$ and $S$, and $L$ more accurate than $S$ with no other differences. The mean absolute error was 2.2 for $R, 2.3$ for $B, 2.4$ for $L, 2.5$ for $C$, and 2.6 for $S$.

There was no effect of location on error, but there was a significant effect of breakpoint on error $\left(F_{2,28}=5.7, p<.01\right)$, with participants being more accurate in $u_{b}=10$ than in $u_{b}=5$. A chart $\times$ breakpoint $\left(F_{8,112}=5.7, p<.0001\right)$ interaction was present. Post-hoc comparisons showed that for $u_{b}=2 R$ had less error than $C$, with no other differences. For $u_{b}=5$ we found that $C, R$, and $B$ had a significantly smaller error than $S$, and also $R$ had less error than $L$. There were no differences between charts for $u_{b}=10$.

Time: There was a significant effect of chart on time $\left(F_{4,56}=7.1\right.$, $p<.0001$ ). Pair-wise means comparison (all $p<.05$ ) showed that $S$ was significantly slower than $R$ and $L$ with no other differences. Mean times were fastest for $R(3.7 \mathrm{~s})$, followed by $L$ (3.8 s), $B(4.4 \mathrm{~s}), C$ $(4.5 \mathrm{~s})$, and $S(5.5 \mathrm{~s})$.

\subsection{Questionnaire Results}

In the questionnaire we asked participants to rank the different charts overall and for each task $\times$ location. The overall ranking is depicted in Fig. 9(d). For each task and task $\times$ location, $S$ was always ranked last and $C$ was always ranked first. $B$ and $L$ switched position depending on location and task, but $B$ was ranked second more often than $L$. The most interesting difference from this trend occurred in the slope task where $L$ shared Rank 1 and 2 with $C$ for the across location and was also ranked second for focus + context .

We also asked participants to report the general confidence in their answer with each chart on a 7-point Likert scale. Overall, only $B$ and $C$ received answers indicating that participants were confident in their answers. For $L$ participants answered to be slightly inconfident to neutral, while for $S$ all participants reported not to be confident about their answers. For the slope tasks where participants' answer accuracy was lowest, each technique also received low confidence scores.

\section{Discussion}

Our results suggest that certain types of Dual-Scale chart representations are more effective on a low perceptual level than others. Our findings follow Cleveland's ranking of different tasks in terms of accuracy (position was most accurate, followed by length and slope) but this relative accuracy was different across charts (Fig. 8).

\subsection{Chart Performance}

We found that among the four Dual-Scale charts the cut-out chart was generally the best for tasks which involved relating information in both focus and context. A strong benefit of the cut-out chart was its enlarged $x$ - and $y$-resolution compared to the other charts. Yet due to its visual discontinuity for the bottom focus region we would have expected larger mean differences to the regular chart. The cut-out chart was also the most liked among all charts in the study and participants named it to be clear and most readable. We hypothesize that seeing what the focus information looked like at the regular scale helped participants to cognitively relate the two spaces.

We partially confirmed $\mathrm{H}_{2}$ in that the regular chart of uniform scale outperformed all others (except for cut-out in the length and position tasks). We found across the board that the superimposed chart performed poorly both in terms of accuracy and time. Participants' feedback from the questionnaire was also clearly against the superimposed chart and it was ranked lowest by all but one participant. Participants called it very confusing and demanding too much concentration or reflection to decipher the non-monotonic and discontinuous nature of the two scales. One participant commented that the different visual markers which were necessary to depict data in this chart were a major reason for his confusion. We already hinted at this problem when discussing the superimposed chart properties in Section 3.6.

The broken and lens charts can be situated somewhere in the middle between cut-out and superimposed charts. They were never significantly different from one another but for some conditions better than superimposed and worse than cut-out. Both charts' transformation functions are monotonic (in contrast to superimposed) and their $T$ is injective, yet, the lens chart bridges the gap between focus and context with a smoothly integrated drop-off region and $T_{\text {lens }}$ is continuous whereas $T_{\text {broken }}$ is not. We had hypothesized that this smooth integration and continuity would be beneficial but we could not confirm $H_{4}$. We did observe though a better performance (not significant) at the position task. It may be that tasks that require long visual tracing (like the position one) may be better served by an undivided visual chart but our results seem to suggest that neither visually integrated displays nor visual continuity are necessary for comparing lengths. This requires further exploration. It also still requires further testing to definitely conclude which Dual-Scale properties are the most relevant to predict chart performance. We found that monotonicity was a beneficial property but more data is needed to derive general conclusions. 


\subsection{Influence of Location, Scale, and Task}

According to our first hypothesis, we expected location and breakpoint to significantly impact the performance of our charts. We only partially confirmed $H_{1}$ in that-with increasing scale difference between focus and context-performance significantly decreased. We did not generally find a significant effect between across and focus + context location. It did not seem to matter whether modulus and stimulus were partially in each location or fully embedded.

For task, we confirmed Cleveland's ranking. The position task generally showed the most significant effects and fastest and most accurate results. For the slope task we had expected stronger results as a change in scale also incurs a significant change in the visual angle of a line connecting two data points. We suspect that our design of this task influenced the results. Our participants did not simply have to judge a visual angle or slope (both are related as pointed out by Cleveland [7]), but like in the other two tasks we asked them to judge the slope in data space as a "rate of change." To simplify the task and reduce overall task time, we had designed each slope to connect two data points offset by 1 data unit. This meant that participants only had to compare the slope's $y$-offset which subsequently boiled down to a length comparison in the undistorted $y$-space. If we had flipped $x$ - and $y$-coordinates for this task we expect to have received results similar or identical to the length task. Finally, it is interesting to note that the superimposed chart still fared the worst for slope, indicating that this chart incurred considerable cognitive overhead. For the cut-out chart, participants seem to have been distracted by the presence of the focus area in the slope task as they were significantly less accurate compared to the regular chart.

\subsection{Experiment Design Trade-Offs}

In the design of our experiment we had to make several main choices which influenced our results and also point to limitations of this study.

(1) We believe that the good performance of the regular chart is a direct consequence of our experiment design. We specifically tested modulus values which were visible in the focus for all breakpoint conditions (including $u_{b}=10$ ). This means that comparing stimulus and modulus was always possible across one scale in the regular chart as well as the top of the cut-out chart. For tasks where data is visible in a common scale, using this common scale for tasks is a benefit and participants took advantage of this fact in the cut-out chart leading to its good performance. No other Dual-Scale chart we tested offered this opportunity. For tasks in which the focus information would be invisible or very small, the regular chart would not fare as well and we expect the cut-out chart and possibly others to outperform it.

(2) We made specific choices in chart design which influenced our results. In contrast to our experiment, common implementations of the cut-out chart (see Fig. 1(a)) include two different scales for the top and bottom $y$-axis, which could negatively influence the performance of this chart for tasks involving slope judgements.

(3) There are many different ways to use superimposed charts, e. g. to compare data which uses different units of measure or which is significantly different in scale. We tested the second case and also designed the chart so that there was no overlap in the units on the top and bottom axis. The results could be different for other design alternatives but we hypothesize that this would not be the case.

(4) We tested a specific gridline and tickmark spacing, set to be the same for all charts and scales. Gridlines are an important help in judging magnitude differences $[7,36]$. Yet, it has to be noted that it is generally common to adjust the gridline spacing depending on $D$ and $D^{\prime}$ and that for Dual-Scale charts gridline spacing could be decided upon separately for $D_{1} / D_{1}^{\prime}$ and $D_{2} / D_{2}^{\prime}$. When the data units between each gridline differ for focus and context, additional indicators are necessary as a reference for the difference in scale. Cleveland [7] proposes small gray indicator bars above the two spaces. The influence of such indicators as well as the presence of data labels will have to be investigated further when gridline spacing differs.

(5) Our experiment explicitly compared non-interactive charts that are often seen in print. Our results may differ in interactive charts, where users can adjust the location and magnification of the focus area.
(6) Finally, this experiment focused on low-level perceptual tasks which compared isolated visual variables. These variables were chosen since they form the basis for perceptually extracting quantitative information from different types of charts [7]. From our results one can hypothesize that charts and tasks which predominantly require comparing positions across two scales would work better than those requiring length and slope comparisons. Yet, Further experiments are necessary to confirm the influence of two scales for tasks on fully drawn DualScale bar-, line-, area-, or other charts.

\section{Implications for Using Dual-Scale Charts}

From the results of our experiment we are able to make recommendations on the use and design of Dual-Scale data charts; we recommend to use the cut-out chart and avoid the superimposed chart type. The cut-out chart fared well in our experiment but has design tradeoffs. It either requires more vertical space or needs to be compressed in $y$ to make it fit into the same drawing space as the other chart alternatives. The technique can be extended relatively easily to more foci (e. g., [17]). The timeline visualization in the Google Charts API [14]) includes one version of the cut-out chart, and the ProtoVis [4] website also contains an example of how to build a simple variant of the chart using this visualization toolkit. Yet, both of these alternatives require a certain amount of coding experience to create.

Broken and lens charts can be good alternatives if one does not have the means to create a cut-out chart. Broken charts can be extended easily as outlined in Section 3, and can also be easily designed using tools such as GnuPlot [35], R [25], and online visualization toolkits. The lens chart also fared relatively well in our study but is less easy to implement (see Section 3). We know of no charting software which currently includes charts which resemble our lens chart. Yet, lens charts have the nice property that regions next to the focus gradually decline in scale, which gives more emphasis to data close to the main region of interest. Due to the ease of implementation of the broken chart we recommend to use this chart as an alternative to the cut-out chart if one has to save display space or cannot easily generate a cut-out chart.

Finally, the superimposed chart has the advantage that, for the same drawing space as the other charts, it allows for maximum display space for focus and context. It can be created relatively easily with Excel, one of the most common charting tools in use, and this is likely why it is quite common in practice. Despite these advantages, we recommend against using this chart due to our study results.

\section{Conclusions}

In this paper, we contribute a thorough discussion of the design space for Dual-Scale data charts as well as a study comparing different alternatives. Dual-Scale data charts are important when regular charts reach the limits of their display resolution due to data with varying densities or degrees-of-interest. We outline a specific set of five basic charts and discuss alternatives and generalizations of each. In our perceptual experiment we tested four Dual-Scale charts with different design properties: a cut-out chart, a broken chart, a lens chart, and a superimposed chart. We found that the cut-out chart-a chart showing full context and a visually offset focus region-generally outperformed the other charts for elementary graphical perception tasks. Both the broken as well as the lens chart are competitive alternatives, with the broken chart being generally easier to implement and create with common charting tools. For this reason we recommend using the broken chart as an alternative to the cut-out chart in practice. Finally, our results discourage the use of the superimposed chart as participants performed worst with this chart and also ranked it lowest in terms of subjective preference. In summary, with this paper we fill a gap in the literature on design recommendations for data charts by specifically looking at Dual-Scale charts, we provide a design space and alternatives for designers, and give recommendations on their use in practice.

\section{ACKNOWLEDGMENTS}

We would like to thank the participants of our study and Tobias Isenberg for his helpful comments on earlier drafts of the article. 


\section{References}

[1] P. André, M. L. Wilson, A. Russell, D. A. Smith, A. Owens, and m. c. schraefel. Continuum: Designing Timelines for Hierarchies, Relationships and Scale. In Proc. UIST, pp. 101-110, New York, 2007. ACM. doi> 10.1145/1294211.1294229

[2] S. Bateman, R. Mandryk, C. Gutwin, A. Genest, D. McDine, and C. Brooks. Useful Junk? The Effects of Visual Embellishment on Comprehension and Memorability of Charts. In Proc. CHI, pp. 2573-2582, New York, 2010. ACM. doi> 10.1145/1753326.1753716

[3] J. Bertin. Semiology of Graphics: Diagrams Networks Maps. The University of Wisconsin Press, Madison, USA, 1983.

[4] M. Bostock and J. Heer. Protovis: A Graphical Toolkit for Visualization. Transactions on Visualization and Computer Graphics, 15(6):1121-1128, Nov./Dec. 2009. doi > 10.1109/TVCG.2009.174

[5] M. S. T. Carpendale. A Framework for Elastic Presentation Space. PhD thesis, Simon Fraser University, March 1999.

[6] S. Carpendale and C. Montagnese. A Framework for Unifying Presentation Space. In Proc. UIST, pp. 61-70, New York, 2001. ACM. doi> 10.1145/502348.502358

[7] W. S. Cleveland. The Elements of Graphing Data. Wadsworth Advanced Book Program, 1985.

[8] W. S. Cleveland and R. McGill. Graphical Perception: Theory, Experimentation, and Application to the Development of Graphical Methods. Journal of the American Statistical Association, 79(387):531-554, Sept. 1984. doi > 10.2307/2288400

[9] A. Cockburn, A. Karlson, and B. B. Bederson. A Review of Overview+Detail, Zooming, and Focus+Context Interfaces. ACM Computing Survey, 41:2:1-2:31, Jan. 2009. doi> 10.1145/1456650.1456652

[10] D. R. Cox. Some Remarks on the Role in Statistics of Graphical Methods. Journal of the Royal Statistical Society, 27(1):4-9, Sept. 1978. doi $>10$. 2307/2346220

[11] S. Few. Dual-Scaled Axes in Graphs-Are They Ever the Best Solution? In Visual Business Intelligence Newsletter. Perceptual Edge, Mar. 2008.

[12] S. N. Friel, F. R. Curcio, and G. W. Bright. Making Sense of Graphs: Critical Factors Influencing Comprehension and Instructional Implications. Journal for Research in Mathematics Education, 32(2):124-158, Mar. 2001. doi> 10.2307/749671

[13] G. W. Furnas. Generalized Fisheye Views. In Proc. CHI, pp. 16-23, New York, 1986. ACM. doi > 10.1145/22339.22342

[14] Google Inc. Google Charts API. Online API description on the website http://code.google.com/apis/visualization/. Visited March 2011.

[15] K. W. Haemer. Double Scales are Dangerous. The American Statistician, 2(3):24, June 1948.

[16] J. Heer and M. Bostock. Crowdsourcing Graphical Perception: Using Mechanical Turk to Assess Visualization Design. In Proc. CHI, pp. 203212, New York, 2010. ACM. doi> 10.1145/1753326.1753357

[17] W. Javed and N. Elmqvist. Stack Zooming for Multi-Focus Interaction in Time-Series Data Visualization. In Proc. PacificVis, pp. 33-40, Los Alamitos, 2010. IEEE Computer Society. doi > 10.1109/PACIFICVIS. 2010.5429613

[18] T. A. Keahey. The Generalized Detail-In-Context Problem. In Proc. InfoVis, pp. 44-51, Los Alamitos, 1998. IEEE Computer Society. doi> 10.1109/INFVIS.1998.729558
[19] S. Kosslyn. Graph Design for the Eye and Mind. Oxford University Press, 2007.

[20] Y. K. Leung and M. D. Apperley. A Review and Taxonomy of DistortionOriented Presentation Techniques. ACM Transactions on ComputerHuman Interaction, 1(2):126-160, June 1994. doi> 10.1145/180171. 180173

[21] J. D. Macklinay, G. G. Robertson, and S. K. Card. The Perspective Wall: Detail and Context Smoothly Integrated. In Proc. CHI, pp. 173-180, New York, 1991. ACM. doi> 10.1145/108844.108870

[22] L. Narens. A Theory of Ratio Magnitude Estimation. Journal of Mathematical Psychology, 40(2):109-129, June 1996. doi> 10.1006/jmps. 1996.0011

[23] E. Noik. Space of Presentation Emphasis Techniques for Visualizing Graphs. In Proc. Graphics Interface, pp. 225-233. Canadian HumanComputer Communications Society, 1994.

[24] E. Pietriga and C. Appert. Sigma Lenses: Focus-Context Transitions Combining Space, Time and Translucence. In Proc. CHI, pp. 1343-1352, New York, 2008. ACM. doi> 10.1145/1357054.1357264

[25] R Development Core Team. R: A Language and Environment for Statistical Computing. Software, R Foundation for Statistical Computing, Vienna, Visited June 2011. http://www.R-project.org.

[26] W.-M. Roth and G. M. Bowen. Professionals Read Graphs: A Semiotic Analysis. Journal for Research in Mathematics Education, 32(2):159194, Mar. 2001. doi> 10.2307/749672

[27] M. Sarkar and M. H. Brown. Graphical Fisheye Views of Graphs. In Proc. CHI, pp. 83-91, New York, 1992. ACM. doi > 10.1145/142750.142763

[28] M. Sarkar, S. Snibbe, O. J. Tversky, and S. P. Reiss. Stretching the Rubber Sheet: A Metaphor for Viewing Large Layouts on Small Screens. In Proc. UIST, pp. 81-91, New York, 1993. ACM. doi> 10.1145/168642.168650

[29] C. F. Schmid. Statistical Graphics: Design Principles and Practices. Krieger Pub Co, 1992.

[30] R. Spence and M. D. Apperley. Data Base Navigation: An Office Environment for the Professional. Behaviour and Information Technology, 1(1):43-54, Jan.-Mar. 1981. doi> 10.1080/01449298208914435

[31] E. R. Tufte. The Visual Display of Quantitative Information. Graphic Press, Cheshire, Connecticut, USA, 2001.

[32] F. B. Viégas, M. Wattenberg, F. van Ham, J. Kriss, and M. McKeon. Many Eyes: A Site for Visualization at Internet Scale. IEEE Transactions on Visualization and Computer Graphics, 12(5):1121-1128, Nov./Dec. 2007. doi $>10.1109 /$ TVCG.2007.70577

[33] C. Ware. Information Visualization - Perception for Design. Morgan Kaufmann Publishers, Amsterdam, $2^{\text {nd }}$ edition, 2004.

[34] D. Wigdor, C. Shen, C. Forlines, and R. Balakrishnan. Perception of Elementary Graphical Elements in Tabletop and Multi-surface Environments. In Proc. CHI, pp. 473-482, New York, 2007. ACM. doi > 10. 1145/1240624.1240701

[35] T. Williams, C. Kelley, et al. gnuplot. gnuplot homepage, Visited June 2011. http://www.gnuplot.info/.

[36] A. Zanella, S. Carpendale, and M. Rounding. On the Effects of Viewing Cues in Comprehending Distortions. In Proc. NordiCHI, pp. 119-128. ACM, 2002. doi > 10.1145/572020.572035 